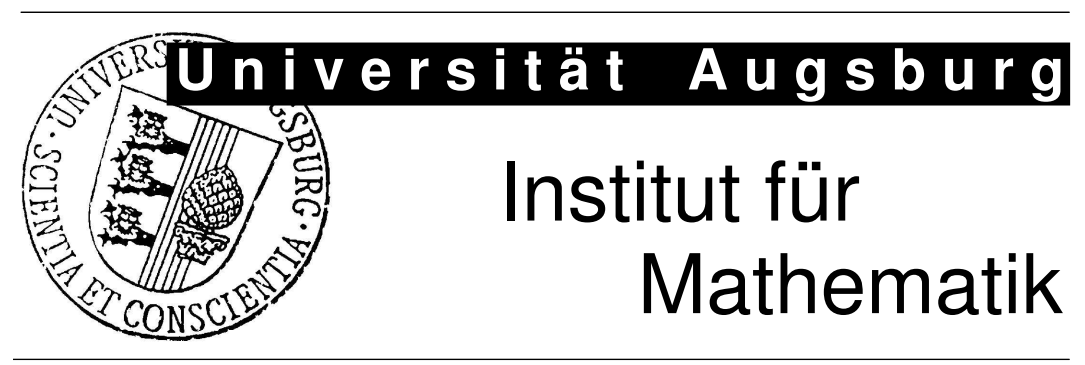

Fritz Colonius, Peter Kloeden, Martin Rasmussen

Morse Spectrum for Nonautonomous Differential Equations

Preprint Nr. 25/2008 - 23. Juni 2008

Institut für Mathematik, Universitätsstraße, D-86 135 Augsburg

http: //www . math. uni-augsburg.de/ 


\section{Impressum:}

Herausgeber:

Institut für Mathematik

Universität Augsburg

86135 Augsburg

http://www . math. uni-augsburg.de/forschung/preprint/

ViSdP:

Martin Rasmussen

Institut für Mathematik

Universität Augsburg

86135 Augsburg

Preprint: Sämtliche Rechte verbleiben den Autoren (C) 2008 


\title{
MORSE SPECTRUM FOR NONAUTONOMOUS DIFFERENTIAL EQUATIONS
}

\author{
Fritz Colonius \\ Department of Mathematics, University of Augsburg, 86135 Augsburg, Germany \\ fritz.colonius@math.uni-augsburg.de \\ Peter E. Kloeden \\ FB Mathematik, Johann-Wolfgang-Goethe-Universität, 60054 Frankfurt, Germany \\ kloeden@math.uni-frankfurt.de \\ Martin Rasmussen \\ Department of Mathematics, University of Augsburg, 86135 Augsburg, Germany \\ martin.rasmussen@math.uni-augsburg.de \\ Received (22 February 2008) \\ Revised (Day Month Year)
}

Dedicated to Ludwig Arnold on the occasion of his 70th birthday

\begin{abstract}
The concept of a Morse decomposition consisting of nonautonomous sets is reviewed for linear cocycle mappings w.r.t. to past, future and all-time convergences. In each case, the set of accumulation points of the finite-time Lyapunov exponents corresponding to points in a nonautonomous set is shown to be an interval. For a finest Morse decomposition, the Morse spectrum is defined as the union of all of the above accumulation point intervals over the different nonautonomous sets in such a finest Morse decomposition. In addition, Morse spectrum is shown to be independent of which finest Morse decomposition is used, when more than one exists.
\end{abstract}

Keywords: Lyapunov exponent, nonautonomous set, Morse decomposition

AMS Subject Classification: 34D08, 37C70

\section{Introduction}

Exponential growth rates for solutions of linear nonautonomous differential equations are a classical topic in the theory of dynamical systems. There are basically two approaches: Either one starts with exponential growth rates and constructs corresponding (generalized) versions of eigenspaces from them; this leads, e.g., to dichotomy spectra and to Oseledet's Theorem. Or one starts with an appropriate generalized version of eigenspaces, and then considers the associated exponential growth rates. In the context of linear flows on vector bundles with compact base space, the latter approach can be based on a topological analysis of the induced 
system on projective space, where, following fundamental work by C. Conley, a finest Morse decomposition can be constructed for systems with a chain transitive base space. This leads to Selgrade's Theorem, proofs of which are given, e.g., by Salamon $\&$ Zehnder ${ }^{11}$ and Bronshte ${ }^{1}{ }^{1}$. This theorem gives a decomposition of the vector bundle into linear subbundles, which, in the autonomous case, reduce to the sums of generalized eigenspaces corresponding to eigenvalues with coinciding real part. Then one can associate to each subbundle a spectral interval of generalized Lyapunov exponents, the Morse spectrum (Colonius $\&$ Kliemann $^{4}$, Grüne ${ }^{6}$ ). Recently, San Martin $\&$ Seco $^{12}$ have extended the Morse spectrum to flows on flag bundles in the context of semi-simple Lie groups.

For nonautonomous differential equations where no compact base space is present, these techniques are not applicable. However, nonautonomous versions of Morse decompositions have recently been derived based on generalizations of pullback attractors (see Rasmussen ${ }^{9,10}$, and see Palmer $\&$ Siegmund $^{7}$ for attractorrepeller pairs). The present paper shows how to define an analogous notion of Morse spectrum in this nonautonomous context. Here the various time domains give rise to different notions, namely past, future and all-time objects.

For the readers' convenience, we have collected some basic notions from nonautonomous dynamics in the ensuing Section 2. Then, in Sections 3 and 4, basic results from Rasmussen ${ }^{9,10}$ are recalled which yield appropriate notions of attractors and repellers leading to finest Morse decompositions for the induced systems on projective space. Section 5 presents the main results of the present paper: a nonautonomous version of Morse spectrum with some insight into its structure. Finally, an example is discussed.

Notation. Given a metric space $(X, d)$, we write $U_{\varepsilon}\left(x_{0}\right)=\left\{x \in X: d\left(x, x_{0}\right)<\varepsilon\right\}$ for the $\varepsilon$-neighborhood of a point $x_{0} \in X$. For arbitrary nonempty sets $A, B \subset X$ and $x \in X$, let $d(x, A):=\inf \{d(x, y): y \in A\}$ be the distance of $x$ to $A$ and $d(A \mid B):=\sup \{d(x, B): x \in A\}$ be the Hausdorff semi-distance of $A$ and $B$.

We denote by $\mathbb{R}^{N \times N}$ the set of all real $N \times N$ matrices. The Euclidean space $\mathbb{R}^{N}$ is equipped with the Euclidean norm $\|\cdot\|$, which is induced by the scalar product $\langle\cdot, \cdot\rangle$, defined by $\langle x, y\rangle:=\sum_{i=1}^{N} x_{i} y_{i}$. To introduce the real projective space $\mathbb{P}^{N-1}$ of $\mathbb{R}^{N}$, we say that two nonzero elements $x, y \in \mathbb{R}^{N}$ are equivalent if there exists a $c \in \mathbb{R}$ such that $x=c y$. The equivalence class of $x \in \mathbb{R}^{N}$ is denoted by $\mathbb{P} x$, and we call the set of all equivalent classes the projective space $\mathbb{P}^{N-1}$. Equipped with the metric $d_{\mathbb{P}}: \mathbb{P}^{N-1} \times \mathbb{P}^{N-1} \rightarrow[0, \sqrt{2}]$, given by

$$
d_{\mathbb{P}}(\mathbb{P} v, \mathbb{P} w)=\min \left\{\left\|\frac{v}{\|v\|}-\frac{w}{\|w\|}\right\|,\left\|\frac{v}{\|v\|}+\frac{w}{\|w\|}\right\|\right\} \quad \text { for all } 0 \neq v, w \in \mathbb{R}^{N}
$$

the projective space is a compact metric space. For any $v \in \mathbb{P}^{N-1}$, we define $\mathbb{P}^{-1} v:=$ $\left\{x \in \mathbb{R}^{N}: \mathbb{P} x=v\right\} \cup\{0\}$. 


\section{Cocycles and Nonautonomous Sets}

Throughout this paper, I denotes a real interval of the form $(-\infty, 0],[0, \infty)$ or $\mathbb{R}$, respectively. Given a metric space $(X, d)$, a cocycle is a mapping $\varphi: \mathbb{I} \times \mathbb{I} \times X \rightarrow X$ with

$$
\varphi(\tau, \tau, \xi)=\xi \quad \text { and } \quad \varphi(t, \tau, \xi)=\varphi(t, s, \varphi(s, \tau, \xi))
$$

for all $\tau, t, s \in \mathbb{I}$ and $\xi \in X$. The set $X$ is called phase space, and $\mathbb{I} \times X$ is called extended phase space. The general solution of a nonautonomous differential equation $\dot{x}=f(t, x)$ is a cocycle if the right hand side $f: \mathbb{R} \times \mathbb{R}^{N} \rightarrow \mathbb{R}^{N}$ satisfies conditions guaranteeing global existence and uniqueness of solutions.

A subset $M$ of the extended phase space $\mathbb{I} \times X$ is called nonautonomous set; we use the term $t$-fiber of $M$ for the set $M(t):=\{x \in X:(t, x) \in M\}$ (which may be empty), $t \in \mathbb{I}$. We call $M$ closed or compact if all $t$-fibers are closed or compact, respectively. Finally, a nonautonomous set $M$ is called invariant (w.r.t. the cocycle $\varphi)$ if $\varphi(t, \tau, M(\tau))=M(\tau+t)$ for all $t, \tau \in \mathbb{I}$.

In case $X=\mathbb{R}^{N}$, a cocycle $\varphi$ is called linear if for given $\alpha, \beta \in \mathbb{R}$, we have

$$
\varphi(t, \tau, \alpha x+\beta y)=\alpha \varphi(t, \tau, x)+\beta \varphi(t, \tau, y) \quad \text { for all } t, \tau \in \mathbb{I} \text { and } x, y \in \mathbb{R}^{N} .
$$

For instance, a linear cocycle is generated by a linear nonautonomous differential equation $\dot{x}=B(t) x$, where $B: \mathbb{I} \rightarrow \mathbb{R}^{N \times N}$ is continuous. Given a linear cocycle $\varphi$, there exists a corresponding matrix-valued function $\Phi: \mathbb{I} \times \mathbb{I} \rightarrow \mathbb{R}^{N \times N}$ with $\Phi(t, \tau) x=\varphi(t, \tau, x)$ for all $t, \tau \in \mathbb{R}$ and $x \in \mathbb{R}^{N}$. We will also use the term linear cocycle for this function. $\Phi$ canonically induces a cocycle $\mathbb{P} \Phi$ on $\mathbb{P}^{N-1}$ by the definition

$$
\mathbb{P} \Phi(t, \tau) \mathbb{P} x:=\mathbb{P}(\Phi(t, \tau) x) \quad \text { for all } t, \tau \in \mathbb{R} \text { and } x \in \mathbb{R}^{N}
$$

(see Colonius \& Kliemann ${ }^{5}$, Lemma 5.2.1, p. 149).

\section{Attractivity and Repulsivity}

In this section, several notions of local attractivity and repulsivity are explained (see also Rasmussen ${ }^{8}$ ). The concepts are introduced for the past (past attractivity and repulsivity), the future (future attractivity and repulsivity) and the entire time (all-time attractivity and repulsivity).

Throughout this section, let $(X, d)$ be a metric space and $\varphi: \mathbb{I} \times \mathbb{I} \times X \rightarrow X$ be a cocycle.

Note that the following notions of attractor are local forms of attractors which have been discussed since the 1990s. For instance, a past attractor is a local form of a pullback attractor (see, e.g., Cheban, Kloeden $\mathcal{E}^{3} S c h m a l f u \beta^{2}$ ), i.e., it attracts a neighborhood of itself in the sense of pullback attraction. Moreover, a future attractor is a local form of a forward attractor, and an all-time attractor is a local form of a uniform attractor as discussed, e.g., in Chepyzhov ${ }^{3}$ Vishik $^{3}$. 
Definition 3.1 (Attractors and repellers). Let $A$ and $R$ be invariant and compact nonautonomous sets.

(i) In case $\mathbb{I}$ is unbounded below, $A$ is called a past attractor if there exists an $\eta>0$ such that

$$
\lim _{t \rightarrow \infty} d\left(\varphi\left(\tau, \tau-t, U_{\eta}(A(\tau-t))\right) \mid A(\tau)\right)=0 \quad \text { for all } \tau \leq 0,
$$

(ii) and $R$ is called a past repeller if there exists an $\eta>0$ such that

$$
\lim _{t \rightarrow \infty} d\left(\varphi\left(\tau-t, \tau, U_{\eta}(R(\tau))\right) \mid R(\tau-t)\right)=0 \quad \text { for all } \tau \leq 0 .
$$

(iii) In case $\mathbb{I}$ is unbounded above, $A$ is called a future attractor if there exists an $\eta>0$ such that

$$
\lim _{t \rightarrow \infty} d\left(\varphi\left(\tau+t, \tau, U_{\eta}(A(\tau))\right) \mid A(\tau+t)\right)=0 \text { for all } \tau \geq 0,
$$

(iv) and $R$ is called a future repeller if there exists an $\eta>0$ such that

$$
\lim _{t \rightarrow \infty} d\left(\varphi\left(\tau, \tau+t, U_{\eta}(R(\tau+t))\right) \mid R(\tau)\right)=0 \quad \text { for all } \tau \geq 0 .
$$

(v) In case $\mathbb{I}=\mathbb{R}, A$ is called an all-time attractor if $A$ is both a past and future attractor, i.e., there exists an $\eta>0$ such that (3.1) and (3.3) hold,

(vi) and $R$ is called an all-time repeller if $R$ is both a past and future repeller, i.e., there exists an $\eta>0$ such that (3.2) and (3.4) hold.

\section{Remark 3.1.}

(i) The notion of an all-time attractor and all-time repeller is slightly different from the concept used in Rasmussen ${ }^{8,10}$, where uniform attraction and repulsion is required, respectively.

(ii) The notions of future attractivity and repulsivity can be derived from the concept of past attractivity and repulsivity via time reversal. A past attractor (repeller, respectively) corresponds to a future repeller (attractor, respectively) of the system under time reversal.

(iii) The Hausdorff semi-distance $d$ in Definition 3.1 can equivalently be replaced by the Hausdorff distance $d_{H}$, which for nonempty sets $A, B \subset X$ is defined by $d_{H}(A, B):=\max \{d(A \mid B), d(B \mid A)\}$.

(iv) Every invariant and compact nonautonomous set of the differential equation $\dot{x}=x$ is a past repeller. Therefore, past repellers are not uniquely determined in general, in contrast to past attractors (see Rasmussen ${ }^{8}$, Proposition 2.37).

Example 3.1. We consider the linear nonautonomous differential equation

$$
\dot{x}=a(t) x
$$

with a continuous function $a: \mathbb{R} \rightarrow \mathbb{R}$. . Then every invariant and compact nonautonomous set $M \subset \mathbb{R} \times \mathbb{R}$ is a 
- past attractor if and only if $\lim _{t \rightarrow-\infty} \int_{t}^{0} a(s) d s=-\infty$,

- past repeller if and only if $\lim _{t \rightarrow-\infty} \int_{t}^{0} a(s) d s=\infty$,

- future attractor if and only if $\lim _{t \rightarrow \infty} \int_{0}^{t} a(s) d s=-\infty$,

- future repeller if and only if $\lim _{t \rightarrow \infty} \int_{0}^{t} a(s) d s=\infty$,

- all-time attractor if and only if $\lim _{t \rightarrow \infty} \max _{\tau \in\{0,-t\}} \int_{\tau}^{\tau+t} a(s) d s=-\infty$,

- all-time repeller if and only if $\lim _{t \rightarrow \infty} \min _{\tau \in\{0,-t\}} \int_{\tau}^{\tau+t} a(s) d s=\infty$.

All relations follow from the representation $\varphi(t, \tau, \xi)=\xi \exp \left(\int_{\tau}^{t} a(s) d s\right)$, which implies $d\left(\varphi\left(t, \tau, U_{\eta}(\{0\})\right) \mid\{0\}\right)=\eta \exp \left(\int_{\tau}^{t} a(s) d s\right)$. Then let $t$ tend to $\infty$ in case of the future, or $\tau$ tend to $-\infty$ in case of the past, or both limits in case of the entire time.

\section{Morse Decomposition}

This section is devoted to a summary of the basic results from Rasmussen ${ }^{9,10}$ concerning the existence of finest nonautonomous Morse decompositions of the projective flow associated with a linear cocycle. Note that concerning the case of all-time Morse decompositions, the results from Rasmussen ${ }^{10}$ need to be adapted, since the definitions of all-time attractivity and repulsivity are not the same (cf. Remark 3.1 (i)). This will be done in a forthcoming paper.

The first step towards a Morse decomposition is the construction of attractorrepeller pairs.

Theorem 4.1 (Attractor-repeller pairs). Let $\Phi: \mathbb{I} \times \mathbb{I} \times \mathbb{R}^{N} \rightarrow \mathbb{R}^{N}$ be a linear cocycle with induced cocycle $\mathbb{P} \Phi$ on projective space. Then the following statements are fulfilled:

(i) Let $\mathbb{I}$ be unbounded below and $R$ be a past repeller of $\mathbb{P} \Phi$, i.e., there exists an $\eta>0$ such that (3.2) holds. Then the nonautonomous set $R^{*}$, defined by

$$
R^{*}(\tau):=\bigcap_{t^{*} \geq 0} \overline{\bigcup_{t \geq t^{*}} \mathbb{P} \Phi(\tau, \tau-t)\left(\mathbb{P}^{N-1} \backslash U_{\eta}(R(\tau-t))\right)} \quad \text { for all } \tau \in \mathbb{I},
$$

is a past attractor, which is maximal outside $R$ in the following sense: Any past attractor $A \supsetneq R^{*}$ has nonempty intersection with $R$. We call $\left(R^{*}, R\right)$ $a$ past attractor-repeller pair.

(ii) Let $\mathbb{I}$ be unbounded above and $A$ be a future attractor of $\mathbb{P} \Phi$, i.e., there exists an $\eta>0$ such that (3.3) holds. Then the nonautonomous set $A^{*}$, defined by

$$
A^{*}(\tau):=\bigcap_{t^{*} \geq 0} \overline{\bigcup_{t \geq t^{*}} \mathbb{P} \Phi(\tau, \tau+t)\left(\mathbb{P}^{N-1} \backslash U_{\eta}(A(\tau+t))\right)} \quad \text { for all } \tau \in \mathbb{I},
$$

is a future repeller, which is maximal outside $A$ in the following sense: Any future repeller $R \supsetneq A^{*}$ has nonempty intersection with $A$. We call $\left(A, A^{*}\right)$ $a$ future attractor-repeller pair. 
(iii) Let $\mathbb{I}=\mathbb{R}$ and $A$ be an all-time attractor of $\mathbb{P} \Phi$. Then the nonautonomous set $A^{*}$, defined by (4.2) is an all-time repeller, which is maximal outside $A$ in the following sense: Any all-time repeller $R \supsetneq A^{*}$ has nonempty intersection with $A$. We call $\left(A, A^{*}\right)$ an all-time attractor-repeller pair.

(iv) Let $\mathbb{I}=\mathbb{R}$ and $R$ be an all-time repeller of $\mathbb{P} \Phi$. Then the nonautonomous set $R^{*}$, defined by (4.1) is an all-time attractor, which is maximal outside $R$ in the following sense: Any all-time attractor $A \supsetneq R^{*}$ has nonempty intersection with $R$. We call $\left(R^{*}, R\right)$ an all-time attractor-repeller pair.

Proof. See Rasmussen ${ }^{9}$, Theorem 4.3, for the past and future case and a forthcoming paper for the entire time (cf. also Rasmussen ${ }^{10}$, Theorem 3.2).

\section{Remark 4.1.}

(i) In general, there is no formalism to obtain a past repeller from a past attractor and to get a future attractor from a future repeller (see Rasmussen ${ }^{9}$, Example 4.4).

(ii) For an all-time attractor $A$, the relation $\left(A^{*}\right)^{*}=A$ is fulfilled, and an alltime repeller $R$ fulfills $\left(R^{*}\right)^{*}=R$ (cf. a forthcoming paper or Rasmussen ${ }^{10}$, Theorem 3.2).

(iii) In Palmer $\&$ Siegmund ${ }^{7}$, so-called generalized attractor-repeller pairs are introduced, which consist of two invariant nonautonomous sets $A$ and $R$ of $\Phi$ whose fibers are linear subspaces of $\mathbb{R}^{N}$ fulfilling the following three conditions:

(a) $A(t) \oplus R(t)=\mathbb{R}^{N}$ for all $t \in \mathbb{R}$,

(b) given $\tau \in \mathbb{R}, 0 \neq \xi \in A(\tau)$ and $0 \neq \eta \in R(\tau)$, we have

$$
\frac{\|\Phi(t, \tau) \eta\|}{\|\Phi(t, \tau) \xi\|} \rightarrow 0 \text { as } t \rightarrow \infty \text { and } \quad \frac{\|\Phi(t, \tau) \xi\|}{\|\Phi(t, \tau) \eta\|} \rightarrow 0 \text { as } t \rightarrow-\infty
$$

(c) the angle between $A(t)$ and $R(t)$ is bounded below by a positive number.

It is easy to see that each all-time attractor-repeller pair forms a generalized attractor-repeller pair.

The notion of an attractor-repeller pair is generalized by the following definition.

Definition 4.1 (Morse decompositions). A set $\left\{M_{1}, M_{2}, \ldots, M_{n}\right\}$ of nonautonomous sets, the so-called Morse sets, is called past (future, all-time, respectively) Morse decomposition of $\mathbb{P} \Phi$ if the representation

$$
M_{i}=A_{i} \cap R_{i-1} \quad \text { for all } i \in\{1, \ldots, n\}
$$

is fulfilled with past (future, all-time, respectively) attractor-repeller pairs $\left(A_{i}, R_{i}\right)$, $i \in\{0, \ldots, n\}$, fulfilling

$$
\emptyset=A_{0} \subsetneq A_{1} \subsetneq \cdots \subsetneq A_{n}=\mathbb{I} \times \mathbb{P}^{N-1}
$$


and

$$
\mathbb{I} \times \mathbb{P}^{N-1}=R_{0} \supsetneq R_{1} \supsetneq \cdots \supsetneq R_{n}=\emptyset .
$$

The following theorem shows that Morse decompositions are crucial for the dynamical behavior of the nonautonomous dynamical system.

Theorem 4.2 (Dynamical properties). The following statements are fulfilled:

(i) Convergence in forward time. Let $\left\{M_{1}, \ldots, M_{n}\right\}$ be a future (all-time, respectively) Morse decomposition of $\mathbb{P} \Phi$. Then for all $(\tau, x) \in \mathbb{I} \times \mathbb{P}^{N-1}$, there exists an $i \in\{1, \ldots, n\}$ with

$$
\lim _{t \rightarrow \infty} d_{\mathbb{P}}\left(\mathbb{P} \Phi(\tau+t, \tau) x, M_{i}(\tau+t)\right)=0 .
$$

(ii) Convergence in backward time. Let $\left\{M_{1}, \ldots, M_{n}\right\}$ be a past (all-time, respectively) Morse decomposition of $\mathbb{P} \Phi$. Then for all $(\tau, x) \in \mathbb{I} \times \mathbb{P}^{N-1}$, there exists an $i \in\{1, \ldots, n\}$ with

$$
\lim _{t \rightarrow \infty} d_{\mathbb{P}}\left(\mathbb{P} \Phi(\tau-t, \tau) x, M_{i}(\tau-t)\right)=0 .
$$

Proof. See Rasmussen ${ }^{9}$, Theorem 8.5, and Rasmussen ${ }^{10}$, Theorem 4.4.

Further convergence results for Morse decompositions can be found in Rasmussen $^{9}$, Theorem 5.6.

We conclude this section by stating a result concerning finest Morse decompositions, which is an analog to the Theorem of Selgrade (see Selgrade ${ }^{13}$ ).

Theorem 4.3 (Finest Morse decomposition). There exists a finest past (future, all-time, respectively) Morse decomposition $\left\{M_{1}, \ldots, M_{n}\right\}$ of $\mathbb{P} \Phi$, i.e., the number of Morse sets of every past (future, all-time, respectively) Morse decomposition is bounded by $n$. Moreover, we have $n \leq N$ and the decomposition

$$
\mathbb{P}^{-1} M_{1}(t) \oplus \cdots \oplus \mathbb{P}^{-1} M_{n}(t)=\mathbb{R}^{N} \quad \text { for all } t \in \mathbb{I} .
$$

Proof. See Rasmussen ${ }^{9}$, Theorem 8.7, for the past and future case and a forthcoming paper for the entire time (cf. also Rasmussen ${ }^{10}$, Theorem 5.1).

Remark 4.2. A finest Morse decomposition for the past and future is not uniquely determined, but one obtains that the Morse sets of two finest Morse decompositions are converging to each other in Hausdorff distance when time tends to the past or future, respectively.

\section{Morse Spectrum}

In this section, we introduce Morse spectra for a linear cocycle $\Phi: \mathbb{I} \times \mathbb{I} \rightarrow \mathbb{R}^{N \times N}$. Fundamental for what follows is the definition of a finite-time exponential growth rate. 
Definition 5.1 (Finite-time exponential growth rate). For any $(\tau, \xi) \in \mathbb{I} \times$ $\mathbb{R}^{N}, \xi \neq 0$, and $T>0$ with $\tau+T \in \mathbb{I}$, we define the finite-time exponential growth rate by

$$
\lambda^{T}(\tau, \xi):=\frac{1}{T} \ln \frac{\|\Phi(\tau+T, \tau) \xi\|}{\|\xi\|} .
$$

Using this concept, we study limits of growth rates which are attained from initial values $(\tau, \xi)$ within a fixed linear and invariant nonautonomous set.

Definition 5.2. Let $M \subset \mathbb{I} \times \mathbb{R}^{N}$ be an invariant nonautonomous set such that the fibers of $M$, i.e., the sets $M(t)$ for $t \in \mathbb{I}$, are linear subspaces of $\mathbb{R}^{N}$.

(i) If $\mathbb{I}$ is unbounded below, the past spectrum of $\Phi$ over $M$ is defined by

$$
\begin{array}{r}
\Sigma^{-}(M):=\left\{\mu \in \overline{\mathbb{R}}: \text { there exist sequences } T_{k} \rightarrow \infty \text { and }\left(\tau_{k}, \xi_{k}\right) \in M\right. \\
\text { with } \left.\tau_{k}, \tau_{k}+T_{k} \leq 0 \text { and } \lim _{k \rightarrow \infty} \lambda^{T_{k}}\left(\tau_{k}, \xi_{k}\right)=\mu\right\} .
\end{array}
$$

(ii) If $\mathbb{I}$ is unbounded above, the future spectrum of $\Phi$ over $M$ is defined by

$$
\begin{array}{r}
\Sigma^{+}(M):=\left\{\mu \in \overline{\mathbb{R}}: \text { there exist sequences } T_{k} \rightarrow \infty \text { and }\left(\tau_{k}, \xi_{k}\right) \in M\right. \\
\text { with } \left.\tau_{k}, \tau_{k}+T_{k} \geq 0 \text { and } \lim _{k \rightarrow \infty} \lambda^{T_{k}}\left(\tau_{k}, \xi_{k}\right)=\mu\right\} .
\end{array}
$$

(iii) If $\mathbb{I}=\mathbb{R}$, the all-time spectrum of $\Phi$ over $M$ is defined by

$$
\begin{gathered}
\Sigma^{ \pm}(M):=\left\{\mu \in \overline{\mathbb{R}}: \text { there exist sequences } T_{k} \rightarrow \infty \text { and }\left(\tau_{k}, \xi_{k}\right) \in M\right. \\
\text { with } \left.\lim _{k \rightarrow \infty} \lambda^{T_{k}}\left(\tau_{k}, \xi_{k}\right)=\mu\right\} .
\end{gathered}
$$

Note that the conditions $\tau_{k}+T_{k} \leq 0$ and $T_{k} \rightarrow \infty$ in (i) imply that $\tau_{k} \rightarrow-\infty$ when $k \rightarrow \infty$. The limits in (i) can thus be seen as pullback limits, whereas we obtain forward limits in (ii) and arbitrary limits in (iii).

In the following, we allow $\pm \infty$ as boundary points of intervals, e.g., $[-\infty, \infty]:=$ $(-\infty, \infty) \cup\{-\infty, \infty\}$ and $[\infty, \infty]:=\{\infty\}$

With these definitions, the past (future, all-time, respectively) spectrum of a linear and invariant nonautonomous set turns out to be a closed interval.

Theorem 5.1. Let $M \subset \mathbb{I} \times \mathbb{R}^{N}$ be an invariant nonautonomous set such that the fibers of $M$ are linear subspaces of $\mathbb{R}^{N}$, and let $\Sigma(M):=\Sigma^{-}(M), \Sigma^{+}(M), \Sigma^{ \pm}(M)$ be the past (future, all-time, respectively) spectrum of $\Phi$ over $M$, respectively. Then $\Sigma(M)$ is nonempty, closed and connected, i.e., there exist $-\infty \leq \sigma_{*} \leq \sigma^{*} \leq \infty$ such that

$$
\Sigma(M)=\left[\sigma_{*}, \sigma^{*}\right]
$$

Proof. The closedness of the spectrum follows directly from the definition via the limits, and the spectrum is nonempty, since the limes superior of the sequence 
$\lambda^{T_{k}}\left(\tau_{k}, \xi_{k}\right)$ for given arbitrary sequences $T_{k} \rightarrow \infty$ and $\left(\tau_{k}, \xi_{k}\right) \in M$ belongs to $\Sigma(M)$. We now choose $s^{-}, s^{+} \in \Sigma(M)$ with $s^{-}<s^{+}$, and let $s \in\left(s^{-}, s^{+}\right)$. Then there exist sequences $T_{k}^{-}, T_{k}^{+} \rightarrow \infty$ and $\left(\tau_{k}^{-}, \xi_{k}^{-}\right),\left(\tau_{k}^{+}, \xi_{k}^{+}\right) \in M$ such that

$$
\lim _{k \rightarrow \infty} \lambda^{T_{k}^{-}}\left(\tau_{k}^{-}, \xi_{k}^{-}\right)=s^{-} \text {and } \lim _{k \rightarrow \infty} \lambda^{T_{k}^{+}}\left(\tau_{k}^{+}, \xi_{k}^{+}\right)=s^{+} .
$$

We can assume that both $\lambda^{T_{k}^{+}}\left(\tau_{k}^{+}, \xi_{k}^{+}\right)>s$ and $\lambda^{T_{k}^{-}}\left(\tau_{k}^{-}, \xi_{k}^{-}\right)<s$ is fulfilled for all $k \in \mathbb{N}$. Given $k \in \mathbb{N}$, the function

$$
c \mapsto \lambda^{c T_{k}^{+}+(1-c) T_{k}^{-}}\left(c \tau_{k}^{+}+(1-c) \tau_{k}^{-}, c \xi_{k}^{+}+(1-c) \xi_{k}^{-}\right), \quad c \in[0,1],
$$

is continuous, and hence, there exists a $c_{k} \in[0,1]$ such that

$$
\lambda^{c_{k} T_{k}^{+}+\left(1-c_{k}\right) T_{k}^{-}}\left(c_{k} \tau_{k}^{+}+\left(1-c_{k}\right) \tau_{k}^{-}, c_{k} \xi_{k}^{+}+\left(1-c_{k}\right) \xi_{k}^{-}\right)=s .
$$

This implies $s \in \Sigma(M)$.

The following notion of Morse spectrum relies on finest Morse decompositions. In the case of the past and future, a finest Morse decomposition is not uniquely determined (see Remark 4.2), but the following lemma says that the kind of nonuniqueness does not affect the spectra over the Morse sets.

Lemma 5.1. Let $\mathcal{M}:=\left\{M_{1}, \ldots, M_{n}\right\}$ and $\tilde{\mathcal{M}}:=\left\{\tilde{M}_{1}, \ldots, \tilde{M}_{n}\right\}$ be finest future Morse decompositions of $\mathbb{P} \Phi$. Then for all $i \in\{1, \ldots, n\}$, we have

$$
\Sigma^{+}\left(\mathbb{P}^{-1} M_{i}\right)=\Sigma^{+}\left(\mathbb{P}^{-1} \tilde{M}_{i}\right) .
$$

A similar statement is fulfilled for finest past Morse decompositions.

Proof. Let $\emptyset=A_{0} \subsetneq A_{1} \subsetneq \cdots \subsetneq A_{n}=\mathbb{I} \times \mathbb{P}^{N-1}$ and $\emptyset=\tilde{A}_{0} \subsetneq \tilde{A}_{1} \subsetneq \cdots \subsetneq \tilde{A}_{n}=$ $\mathbb{I} \times \mathbb{P}^{N-1}$ be the future attractor sequences leading to $\mathcal{M}$ and $\tilde{\mathcal{M}}$, respectively. Note that we have $A_{j}^{*}=\tilde{A}_{j}^{*}$ for $j \in\{1, \ldots, n\}$ (see Rasmussen ${ }^{9}$, Theorem 8.7). Let $i \in\{1, \ldots, n\}$ and $\mu \in \Sigma^{+}\left(\mathbb{P}^{-1} M_{i}\right)$, i.e., there exist sequences $T_{k} \rightarrow \infty$ and $\left(\tau_{k}, \xi_{k}\right) \in$ $\mathbb{P}^{-1} M_{i}$ with $\tau_{k}, \tau_{k}+T_{k} \geq 0$ and $\lim _{k \rightarrow \infty} \lambda^{T_{k}}\left(\tau_{k}, \xi_{k}\right)=\mu$. We denote the projection on $\mathbb{P}^{-1} \tilde{M}_{i}(t)$ with null space $\mathbb{P}^{-1} \tilde{M}_{1}(t) \oplus \cdots \oplus \mathbb{P}^{-1} \tilde{M}_{i-1}(t) \oplus \mathbb{P}^{-1} \tilde{M}_{i+1}(t) \oplus \mathbb{P}^{-1} \tilde{M}_{n}(t)$ by $P(t), t \in \mathbb{I}$, and we define

$$
\tilde{\xi}_{k}:=P\left(\tau_{k}\right) \xi_{k} \quad \text { and } \quad \eta_{k}:=\xi_{k}-\tilde{\xi}_{k} \text { for all } k \in \mathbb{N} .
$$

We have $M_{i}(t), \tilde{M}_{i}(t) \subset A_{i-1}^{*}(t)=\tilde{A}_{i-1}^{*}(t)=\mathbb{P}\left(\mathbb{P}^{-1} \tilde{M}_{i}(t) \oplus \cdots \oplus \mathbb{P}^{-1} \tilde{M}_{n}(t)\right)$ for $t \in \mathbb{I}$ (note that the last equality follows from the proof of Rasmussen ${ }^{9}$, Theorem 8.7), and this implies

$$
\eta_{k} \in \mathbb{P}^{-1} \tilde{M}_{i+1}\left(\tau_{k}\right) \oplus \cdots \oplus \mathbb{P}^{-1} \tilde{M}_{n}\left(\tau_{k}\right) \text { for all } k \in \mathbb{N},
$$

since $P\left(\tau_{k}\right) \eta_{k}=0$. Let $Q(t), t \in \mathbb{I}$, denote the projection on $\mathbb{P}^{-1} \tilde{M}_{1}(t) \oplus \cdots \oplus$ $\mathbb{P}^{-1} \tilde{M}_{i}(t)$ with null space $\mathbb{P}^{-1} \tilde{M}_{i+1}(t) \oplus \cdots \oplus \mathbb{P}^{-1} \tilde{M}_{n}(t)$. By (5.8), we have

$$
\tilde{\xi}_{k}=P\left(\tau_{k}\right) \xi_{k}=Q\left(\tau_{k}\right) \xi_{k} \quad \text { for all } k \in \mathbb{N} .
$$


Moreover, the projected null space of $Q$, i.e., the set $\mathbb{P}\left(\mathbb{P}^{-1} \tilde{M}_{i+1} \oplus \cdots \oplus \mathbb{P}^{-1} \tilde{M}_{n}\right)=$ $\mathbb{P}\left(\mathbb{P}^{-1} M_{i+1} \oplus \cdots \oplus \mathbb{P}^{-1} M_{n}\right)$, is a future repeller, and the projected range of $Q$, i.e., the set $\mathbb{P}\left(\mathbb{P}^{-1} M_{1} \oplus \cdots \oplus \mathbb{P}^{-1} M_{i}\right)$, is the corresponding future attractor. This means that the projected range and null space of $Q(t)$ are separated uniformly for all $t \in \mathbb{I}$ (see Rasmussen ${ }^{8}$, Theorem 3.5 (i)), and hence, we have a $K_{1}>1$ such that $\|Q(\tau) \xi\| \leq K_{1}\|\xi\|$ for all $\tau \in \mathbb{I}$ and $\xi \in \mathbb{R}^{N}$. Furthermore, the set $\mathbb{P}^{-1} M_{i}(t)$ is also separated from the projected null space of $Q$ uniformly in $t \in \mathbb{I}$, which is given by $\mathbb{P}\left(\mathbb{P}^{-1} \tilde{M}_{i+1} \oplus \cdots \oplus \mathbb{P}^{-1} \tilde{M}_{n}\right)(t)=\mathbb{P}\left(\mathbb{P}^{-1} M_{i+1} \oplus \cdots \oplus \mathbb{P}^{-1} M_{n}\right)(t)$ (note that $\mathbb{P}\left(\mathbb{P}^{-1} M_{1} \oplus \cdots \oplus \mathbb{P}^{-1} M_{i}\right)$ and $\mathbb{P}\left(\mathbb{P}^{-1} \tilde{M}_{i+1} \oplus \cdots \oplus \mathbb{P}^{-1} \tilde{M}_{n}\right)=\mathbb{P}\left(\mathbb{P}^{-1} M_{i+1} \oplus\right.$ $\left.\cdots \oplus \mathbb{P}^{-1} M_{n}\right)$ are a future attractor-repeller pair), and thus, there exists a constant $K_{2}>1$ with

$$
\|P(\tau) \xi\| \stackrel{(*)}{=}\|Q(\tau) \xi\| \geq \frac{1}{K_{2}}\|\xi\| \quad \text { for all }(\tau, \xi) \in \mathbb{P}^{-1} M_{i} .
$$

Note that $(*)$ follows as in $(5.9)$, where $\left(\tau_{k}, \xi_{k}\right) \in \mathbb{P}^{-1} M_{i}$. We obtain

$$
\begin{aligned}
\lambda^{T_{k}}\left(\tau_{k}, \tilde{\xi}_{k}\right) & =\frac{1}{T_{k}} \ln \left(\frac{\left\|\Phi\left(\tau_{k}+T_{k}, \tau_{k}\right) P\left(\tau_{k}\right) \xi_{k}\right\|}{\left\|P\left(\tau_{k}\right) \xi_{k}\right\|}\right) \\
& =\frac{1}{T_{k}} \ln \left(\frac{\left\|P\left(\tau_{k}+T_{k}\right) \Phi\left(\tau_{k}+T_{k}, \tau_{k}\right) \xi_{k}\right\|}{\left\|P\left(\tau_{k}\right) \xi_{k}\right\|}\right) \\
& \leq \frac{1}{T_{k}} \ln \left(K_{1} K_{2} \frac{\left\|\Phi\left(\tau_{k}+T_{k}, \tau_{k}\right) \xi_{k}\right\|}{\left\|\xi_{k}\right\|}\right)
\end{aligned}
$$

(we again used the fact that $Q(t)$ and $P(t)$ coincide on $\mathbb{P}^{-1} M_{i}(t)$ ). In addition, we have

$$
\begin{aligned}
\lambda^{T_{k}}\left(\tau_{k}, \tilde{\xi}_{k}\right) & =\frac{1}{T_{k}} \ln \left(\frac{\left\|P\left(\tau_{k}+T_{k}\right) \Phi\left(\tau_{k}+T_{k}, \tau_{k}\right) \xi_{k}\right\|}{\left\|P\left(\tau_{k}\right) \xi_{k}\right\|}\right) \\
& \geq \frac{1}{T_{k}} \ln \left(\frac{\left\|\Phi\left(\tau_{k}+T_{k}, \tau_{k}\right) \xi_{k}\right\|}{K_{1} K_{2}\left\|\xi_{k}\right\|}\right),
\end{aligned}
$$

and we obtain $\mu \in \Sigma^{+}\left(\tilde{M}_{i}\right)$. This finishes the proof of this lemma.

The following definition of a Morse spectrum builds upon finest Morse decompositions. In fact, the Morse spectrum is defined as the union of the spectra of the corresponding Morse sets.

Definition 5.3 (Morse spectrum). Let $\left\{M_{1}, \ldots, M_{n}\right\}$ be a finest past (future, all-time, respectively) Morse decomposition of $\mathbb{P} \Phi$ from Theorem 4.3. Then the past (future, all-time, respectively) Morse spectrum of $\Phi$ is defined by

$$
\Sigma(\Phi):=\bigcup_{i=1}^{n} \Sigma\left(\mathbb{P}^{-1} M_{i}\right)
$$

where $\Sigma\left(M_{i}\right)$ denotes the past (future, all-time) spectrum of $\Phi$ over $M_{i}, i=1, \ldots, n$.

The following theorem says that the Morse spectra contain all Lyapunov exponents of the system. 
Theorem 5.2 (Lyapunov exponents are contained in the Morse spectrum). Let $(\tau, \xi) \in \mathbb{I} \times \mathbb{R}^{N}$ such that

$$
\mu=\lim _{k \rightarrow \infty} \frac{1}{T_{k}} \ln \left\|\Phi\left(\tau+T_{k}, \tau\right) \xi\right\|
$$

for some $\mu \in \overline{\mathbb{R}}$ and some sequence $\left\{T_{k}\right\}_{k \in \mathbb{N}}$ with $\lim _{k \rightarrow \infty} T_{k}=\infty$. Then $\mu \in$ $\Sigma^{+}(\Phi) \subset \Sigma^{ \pm}(\Phi)$. Moreover, let $(\tau, \xi) \in \mathbb{I} \times \mathbb{R}^{N}$ such that

$$
\mu=\lim _{k \rightarrow \infty} \frac{1}{T_{k}} \ln \left\|\Phi\left(\tau-T_{k}, \tau\right) \xi\right\|
$$

for some $\mu \in \overline{\mathbb{R}}$ and some sequence $\left\{T_{k}\right\}_{k \in \mathbb{N}}$ with $\lim _{k \rightarrow \infty} T_{k}=\infty$. Then $-\mu \in$ $\Sigma^{-}(\Phi) \subset \Sigma^{ \pm}(\Phi)$.

Proof. Let $\left\{M_{1}, \ldots, M_{n}\right\}$ be some finest future Morse decomposition. We write $\xi=m_{1}+\cdots+m_{n}$ with $m_{i} \in \mathbb{P}^{-1} M_{i}(\tau), i \in\{1, \ldots, n\}$, and we choose $j \in\{1, \ldots, n\}$ minimal with $m_{j} \neq 0$. Then Rasmussen ${ }^{8}$, Proposition 3.19, implies that

$$
\lim _{k \rightarrow \infty} \frac{\left\|\Phi\left(\tau+T_{k}, \tau\right) m_{i}\right\|}{\left\|\Phi\left(\tau+T_{k}, \tau\right) m_{j}\right\|}=0 \quad \text { for all } i \in\{j+1, \ldots, n\}
$$

(note that $\mathbb{P}\left(\mathbb{P}^{-1} M_{1} \oplus \cdots \oplus \mathbb{P}^{-1} M_{j}\right)$ is a future attractor). In addition, we obtain

$$
\begin{aligned}
\left\|\Phi\left(\tau+T_{k}, \tau\right) \xi\right\| & \leq \sum_{i=j}^{n}\left\|\Phi\left(\tau+T_{k}, \tau\right) m_{i}\right\| \\
& =\left\|\Phi\left(\tau+T_{k}, \tau\right) m_{j}\right\| \sum_{i=j}^{n} \frac{\left\|\Phi\left(\tau+T_{k}, \tau\right) m_{i}\right\|}{\left\|\Phi\left(\tau+T_{k}, \tau\right) m_{j}\right\|} \\
& =\left\|\Phi\left(\tau+T_{k}, \tau\right) m_{j}\right\|\left(1+\sum_{i=j+1}^{n} \frac{\left\|\Phi\left(\tau+T_{k}, \tau\right) m_{i}\right\|}{\left\|\Phi\left(\tau+T_{k}, \tau\right) m_{j}\right\|}\right)
\end{aligned}
$$

and

$$
\begin{aligned}
\left\|\Phi\left(\tau+T_{k}, \tau\right) \xi\right\| & \geq\left|\left\|\Phi\left(\tau+T_{k}, \tau\right) m_{j}\right\|-\left\|\sum_{i=j+1}^{n} \Phi\left(\tau+T_{k}, \tau\right) m_{i}\right\|\right| \\
& =\left\|\Phi\left(\tau+T_{k}, \tau\right) m_{j}\right\|\left|1-\left\|\sum_{i=j+1}^{n} \frac{\Phi\left(\tau+T_{k}, \tau\right) m_{i}}{\left\|\Phi\left(\tau+T_{k}, \tau\right) m_{j}\right\|}\right\|\right| .
\end{aligned}
$$

Hence, (5.14) implies that

$$
\begin{aligned}
\mu & =\lim _{k \rightarrow \infty} \frac{1}{T_{k}} \ln \left\|\Phi\left(\tau+T_{k}, \tau\right) \xi\right\|=\lim _{k \rightarrow \infty} \frac{1}{T_{k}} \ln \left\|\Phi\left(\tau+T_{k}, \tau\right) m_{j}\right\| \\
& =\lim _{k \rightarrow \infty} \frac{1}{T_{k}} \ln \frac{\left\|\Phi\left(\tau+T_{k}, \tau\right) m_{j}\right\|}{\left\|m_{j}\right\|} .
\end{aligned}
$$

The second statement of this theorem can be proved similarly. 


\section{An Example}

The following example shows that the intervals of the Morse spectrum need not be disjoint. It is a modification of Salamon $\mathcal{E}$ Zehnder ${ }^{11}$, Example 2.14.

Example 6.1. Consider the differential equation

$$
\dot{x}=\cos (\zeta(t)) x, \quad \dot{y}=(\alpha+\cos (\zeta(t)) y,
$$

where $\alpha>0$ is a parameter and $\zeta(t)$ is the solution of $\dot{\zeta}(t)=\sin \zeta(t), \zeta(0)=\pi / 2$. Then $\zeta(t) \dot{\zeta}(t)>0$ for all $t \in \mathbb{R}$ and $\zeta(t) \rightarrow 0$ for $t \rightarrow-\infty$ and $\zeta(t) \rightarrow \pi$ for $t \rightarrow+\infty$. The sets

$$
\mathcal{V}_{1}:=\mathbb{R} \times \mathbb{R} \times\{0\} \quad \text { and } \quad \mathcal{V}_{2}:=\mathbb{R} \times\{0\} \times \mathbb{R}
$$

are invariant nonautonomous sets with linear fibers. For the projectivized flow, the set $M_{1}(t):=\mathbb{P} \mathcal{V}_{1}(t)$ is an all-time attractor and $M_{2}(t):=\mathbb{P} \mathcal{V}_{2}(t)$ is its complementary repeller. They form a finest all-time Morse decomposition. It follows from (6.1) that

$$
\Sigma^{ \pm}\left(M_{1}\right)=[-1,1] \quad \text { and } \quad \Sigma^{ \pm}\left(M_{2}\right)=[\alpha-1, \alpha+1] .
$$

Hence, for $\alpha \in(0,2]$, these spectral intervals intersect.

Acknowledgement. The authors wish to thank the two anonymous referees for valuable suggestions leading to an improvement of this paper.

\section{References}

1. I. U. Bronshtern, Nonautonomous Dynamical Systems, Shtiintsa, Kishinev, Moldavia, 1984.

2. D. N. Cheban, P. E. Kloeden, and B. Schmalfuß, Pullback attractors in dissipative nonautonomous differential equations under discretization, Journal of Dynamics and Differential Equations 13 (2001), no. 1, 185-213.

3. V. V. Chepyzhov and M. I. Vishik, Attractors for Equations of Mathematical Physics, Colloquium Publications, vol. 49, American Mathematical Society, Providence, Rhode Island, 2002.

4. F. Colonius and W. Kliemann, The Morse spectrum of linear flows on vector bundles, Transactions of the American Mathematical Society 348 (1996), no. 11, 4355-4388.

5. F. Colonius and W. Kliemann, The Dynamics of Control, Birkhäuser, 2000.

6. L. Grüne, A uniform exponential spectrum for linear flows on vector bundles, Journal of Dynamics and Differential Equations 12 (2000), no. 2, 435-448.

7. K. Palmer and S. Siegmund, Generalized attractor-repeller pairs, diagonalizability and integral separation, Advanced Nonlinear Studies 4 (2004), 189-207.

8. M. Rasmussen, Attractivity and Bifurcation for Nonautonomous Dynamical Systems, Springer Lecture Notes in Mathematics, vol. 1907, Springer, Berlin, Heidelberg, New York, 2007.

9. Morse decompositions of nonautonomous dynamical systems, Transactions of the American Mathematical Society 359 (2007), no. 10, 5091-5115.

10. _ All-time Morse decompositions of linear nonautonomous dynamical systems, Proceedings of the American Mathematical Society 136 (2008), no. 3, 1045-1055. 
11. D. Salamon and E. Zehnder, Flows on vector bundles and hyperbolic sets, Transactions of the American Mathematical Society 306 (1988), no. 2, 623-649.

12. L. A. B. San Martin and L. Seco, Morse and Lyapunov spectra and dynamics on flag bundles, Instituto de Matematica, Universidade Estadual de Campinas, Campinas, Brazil, Preprint, 2007.

13. J. F. Selgrade, Isolated invariant sets for flows on vector bundles, Transactions of the American Mathematical Society 203 (1975), 359-390. 